\title{
STRUCTURAL MODIFICATION OF GRAPHENE ON COPPER SUBSTRATES IRRADIATED BY NANOSECOND HIGH-INTENSITY ION BEAMS
}

\author{
O. G. Poddubskaya, ${ }^{1}$ P. P. Kuzhir, ${ }^{1,2}$ A. V. Stepanov, ${ }^{2}$
}

UDC 539.12.04, 53.096 A. A. Martynenko, ${ }^{2}$ and G. E. Remnev ${ }^{2}$

Interaction of a nanosecond high-intensity pulsed ion beam with thin graphene films on copper substrates is analyzed. Methods of Raman spectroscopy are used to investigate the degree of graphene degradation depending on the integral implanted dose. The role of the substrate in the structural degradation of graphene irradiated by charged particle beams is demonstrated using the software package SRIM, intended for modeling radiative defect cascades under irradiation by charged particle beams, and the data on radiation resistance of graphene available in the literature.

Keywords: graphene, high-intensity ion beams, electromagnetic radiation.

\section{INTRODUCTION}

Graphene - a 2D carbon structure - attracts attention of scientific community for solving fundamental problems and practical application since its discovery [1]. Such unique graphene properties as chemical inertness, elasticity (the Young modulus is about $1 \mathrm{TPa}$ [2]), high electrical conductivity (electron mobility $\sim 10^{5} \mathrm{~cm}^{2} \cdot \mathrm{V}^{-1} \cdot \mathrm{s}^{-1}$ [3]), and heat conductivity $\left(5000 \mathrm{~W} \cdot \mathrm{m}^{-1} \cdot \mathrm{K}^{-1}[4]\right)$ in combination with optical transparency $(97 \%)$ and atomic thickness make this material attractive for the development of flexible displays, light-emitting diodes, protective elements, and components of high-frequency electronics. The unique electronic structure of graphene and, in particular, the ability to control highfrequency radiation without significant losses allow graphene to be considered as one of the perspective materials for the design on its basis of optoelectronic on-chip components in intensively developing terahertz (THz) frequency range (0.1-10 THz), in particular, for data transfer and communication systems [5]. Moreover, because of high absorption of $\mathrm{THz}$ radiation in the atmosphere, this channel will be mainly used for data exchange in space, that is, under conditions of enhanced radiative background. Proceeding from the foregoing, investigation of the radiative resistance of graphene and possibility of modification of its properties under irradiation with charged particle beams are urgent problems [6] whose solution receives little attention nowadays.

Because of the atomic graphene thickness, the effects arising during its interaction with charged particles differ significantly from those proceeding in the bulk of the material. The most obvious is the difference between the mechanisms of defect formation caused by the absence of the formed recoil atom cascades and the low probability of migration of neighboring carbon atoms in the 2D structure [7]. Theoretically, the problem of graphene interaction with ionizing radiation was considered in $[8,9]$. Experimental works devoted to the defect formation and radiation resistance of $2 \mathrm{D}$ materials, including graphene, are small in number. In some works (for example, see [7, 10]), the role of the substrate in the formation of defects in graphene during interaction with charged particle beams was pointed out, but

${ }^{1}$ Research Institute for Nuclear Problems of Belarusian State University, Minsk, Belarus, e-mail: paddubskaya@gmail.com; polina.kuzhir@gmail.com; ${ }^{2}$ National Research Tomsk Polytechnic University, Tomsk, Russia, e-mail: stepanovav@mail.ru; martynenkoaa@tpu.ru; remnev06@mail.ru. Translated from Izvestiya Vysshikh Uchebnykh Zavedenii, Fizika, No. 8, pp. 67-72, August, 2018. Original article submitted May 14, 2018. 
because of the complexity of taking into account the graphene transfer effects, in the literature there are no unambiguous answers regarding the influence of substrates of different types.

The present work is devoted to investigation of the influence of a copper substrate on the radiation tolerance of graphene. The methods of Raman spectroscopy are used to analyze the chemical vapor deposited (CVD) graphene films modified under irradiation with carbon $\left(\mathrm{C}^{2+}\right.$ and $\left.\mathrm{C}^{+}\right)$and hydrogen ion $\left(\mathrm{H}^{+}\right)$beams. Since the thin film properties were analyzed directly on copper substrates used in the stage of synthesis, additional effects of graphene transfer can be neglected.

\section{HIGH-INTENSITY SYNTHESIS OF THIN GRAPHENE FILMS AND THEIR IRRADIATION WITH POWERFUL PULSED ION BEAMS (PIB)}

In this work, graphene prepared by CVD on metal substrates was investigated. As a substrate, the copper foil (Alfa Aesar, Germany, with purity of $99.8 \%$ ) $25 \mu \mathrm{m}$ thick with sufficiently smooth surface was used without additional polishing. The copper substrate with sizes $5 \times 5 \mathrm{~cm}$ was put in the first stage into the heating furnace chamber representing a quartz tube $80 \mathrm{~mm}$ in diameter $1 \mathrm{~m}$ long. The entire system was pumped down to pressure of 0.06 mbar, the vacuum system was purged by a nitrogen and hydrogen mixture (pressure in the chamber in the given stage was of the order of $0.40 \mathrm{mbar}$ ) for $1 \mathrm{~h}$ for additional purification and then was heated in a hydrogen flow $\left(60 \mathrm{~cm}^{3} / \mathrm{min}\right.$ and 0.30 mbar) with a rate of $10^{\circ} \mathrm{C} / \mathrm{min}$ to temperature of $1000^{\circ} \mathrm{C}$. Additional annealing of the copper substrate in a hydrogen atmosphere (in some papers, the $\mathrm{H}_{2} / \mathrm{Ar}$ mixture was used) led to surface smoothing, removal of oxide films, and recrystallization and growth of copper grains. In this case, as indicated in [11], the variation of the annealing time allows the graphene grain size and, as a result, its electromagnetic properties to be changed. In the stage of direct synthesis in the chamber for $2 \mathrm{~h}$, methane $\left(\mathrm{CH}_{4} ; 40 \mathrm{~cm}^{3} / \mathrm{min}\right)$ was supplied along with hydrogen $\left(60 \mathrm{~cm}^{3} / \mathrm{min}\right)$; then the entire system was cooled in a static hydrogen atmosphere (pressure in the chamber was 2 mbar in this stage).

The structural properties of the prepared samples (the number of graphene layers and the presence of multilayered graphene islets) as well as its modification under PIB irradiation were studied by Raman spectroscopy. To record the spectra, we used a Nanofinder He (LOTIS TII, Belarus-Japan) confocal microscope with laser excitation wavelength of $473 \mathrm{~nm}$. To decrease the probability of forming additional defects upon exposure to laser radiation, the average radiation power incident on the sample did not exceed $200-400 \mu \mathrm{W}$. The radiation spot size on the sample was $\sim 1 \mu \mathrm{m}(50 \times$ and $100 \times$ objectives were used).

The mechanisms of PIB interaction with thin graphene films were investigated using a TEMP-type accelerator. A beam in the experimental setup was formed by a family of magnetically insulated diodes (MID). Figure $1 a$ shows waveforms of the current, ion diode voltage, and ion current density in the place of sample location in the setup. During experiment, the maximal current density on the target was $\sim 10 \mathrm{~A} / \mathrm{cm}^{2}$, the beam cross sectional area was of the order of $4 \times 4 \mathrm{~cm}^{2}$ in the focal region that was suffice for uniform irradiation of the examined samples. The beam comprised $30 \%$ of hydrogen ions and $70 \%$ of carbon ions. The pulse duration at half maximum was about $80 \mathrm{~ns}$, and the ion density was $1.5 \cdot 10^{12}$ ions $/ \mathrm{cm}^{2}$. The average ion energy was about $290 \mathrm{keV}$. The accelerator was equipped by a vacuum system was based on diffusion and turbomolecular pumps that, in turn, allows the vacuum at a level of $\sim 10^{-5}$ Torr to be obtained.

The samples of CVD graphene on copper substrates were irradiated as follows (Fig. 1b): immediately after synthesis, the initial CVD graphene sample was subdivided into several parts, after which a part of the sample was covered by a copper plate that provided its screening from the PIB, whereas its other part was irradiated. Displacing the copper screen, the area of the open part was gradually increased, which allowed us to separate accurately the areas of one sample with different absorbed doses. It is important to note that in each stage, irradiation was performed by a series of two successive pulses. The period between pulses was $\sim 2-3 \mathrm{~s}$. 

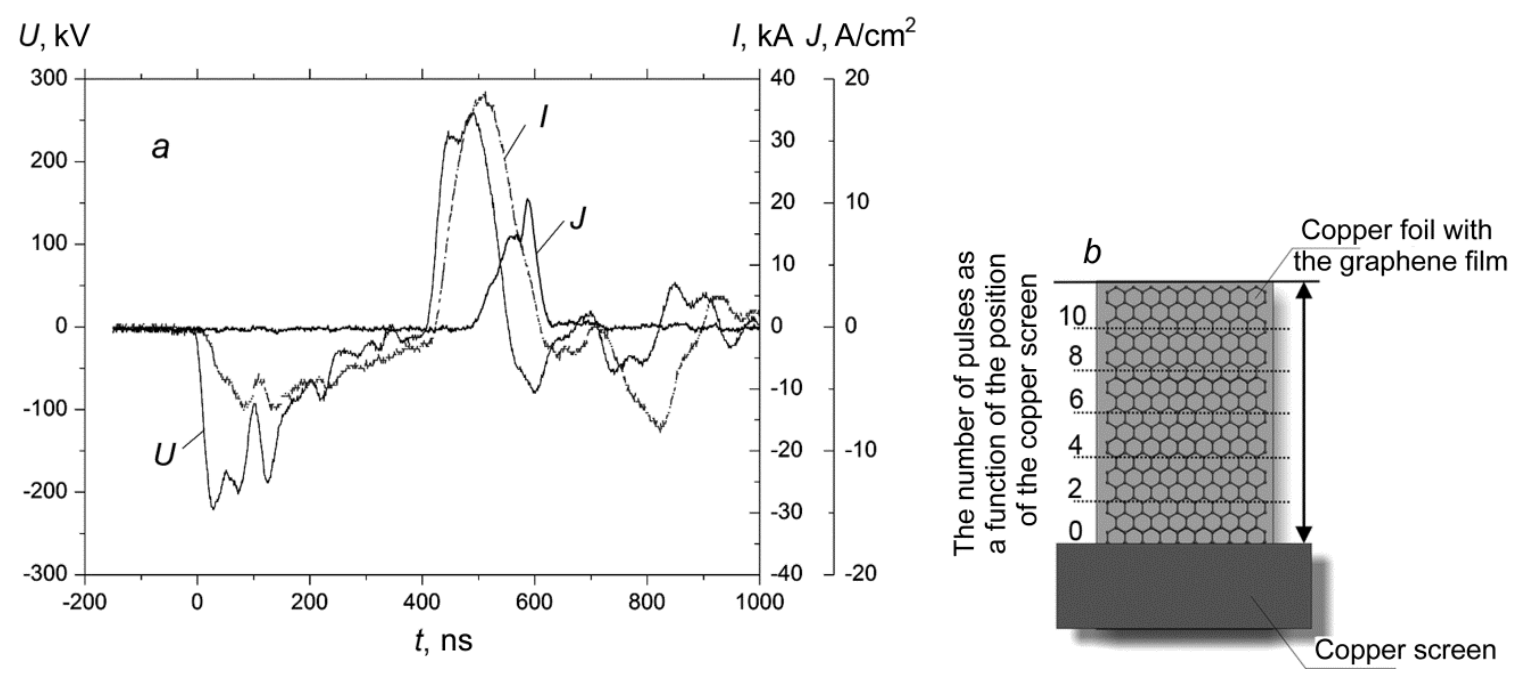

Fig. 1. Waveforms of the current and voltage of the ion diode and of the ion current density. The voltage and the current were measured by built-in sensors of the accelerator in the region of location of the ion diode $(a)$. The ion current density was measured in the region of the sample localization $(b)$. Schematic representation of the procedure of sample irradiation $(c)$.

\section{RESULTS AND DISCUSSION}

Figure 2 shows the Raman spectra of the graphene film on the copper substrate depending on the number of pulses (the average absorbed dose). As is known from the literature [12], some characteristic lines can be identified in the Raman spectrum of graphene that can be analyzed to retrieve important information on the structural properties of the carbon film. Thus, among the main graphene modes are the following modes: the G mode $\left(\sim 1580 \mathrm{~cm}-{ }^{1}\right)$ attributed to oscillations of $s p^{2}$ carbon atoms along the graphene planes (degenerate $E_{2 g}$ symmetry phonon modes); the 2D mode $\left(\sim 2748 \mathrm{~cm}^{-1}\right.$; the position of the given mode depends on the excitation energy) attributed to two-photon resonance whose halfwidth is an important indicator of the number of graphene layers; and the D mode $\left(\sim 1362 \mathrm{~cm}^{-1}\right)$ attributed to breathing oscillations of the six-atom ring in the graphene crystal structure activated when the symmetry of the graphene lattice is broken. It is important to note that initially the carbon film prepared by the CVD method is polycrystalline - consisted of graphene grains separated by defect areas (grain boundaries) and rotated relative to each other. Using the procedure described in [13], it can be demonstrated that the graphene grain sizes in our case were $\sim 20$ $40 \mu \mathrm{m}$.

To estimate the number of graphene layers, two main parameters were used: the ratio of the 2D- and G-mode intensities and the full width at half maximum (FWHM) of the 2D mode [14]. We note that unlike the position and intensity, the FWHM of the 2D mode is less sensitive to the excitation energy and the doping level of graphene. From the analysis of the Raman spectra of thin graphene films before irradiation (Fig. 2b), we can conclude that the large part of our samples is formed by graphene monolayer (FWHM $\sim 34 \mathrm{~cm}^{-1}$ ) with small islets of multilayer structure (FWHM $\sim 49 \mathrm{~cm}^{-1}$ ), which correspond to two or three layers). The weak D mode of the initial sample was caused by defects formed in the growth stage. After irradiation, the ratio of the intensities of the D and G modes $\left(I_{\mathrm{D}} / I_{\mathrm{G}}\right)$ first increased depending on the integral implanted dose during our experiment (to a certain threshold corresponding to 6-8 pulses $\left(\sim 9 \cdot 10^{12}-12 \cdot 10^{12}\right.$ atoms $\left.\left./ \mathrm{cm}^{2}\right)\right)$. Such effect can be associated with the increase in the number of defects in the graphene structure. Further decrease of the intensity of the $G$ mode and simultaneous occurrence of the $\mathrm{D}^{\prime}$ mode $\left(\sim 1620 \mathrm{~cm}^{-1}\right)$ indicate the destruction of the graphene structure and transition to the amorphous phase (Fig. $2 c$ ) that, in turn, leads to 

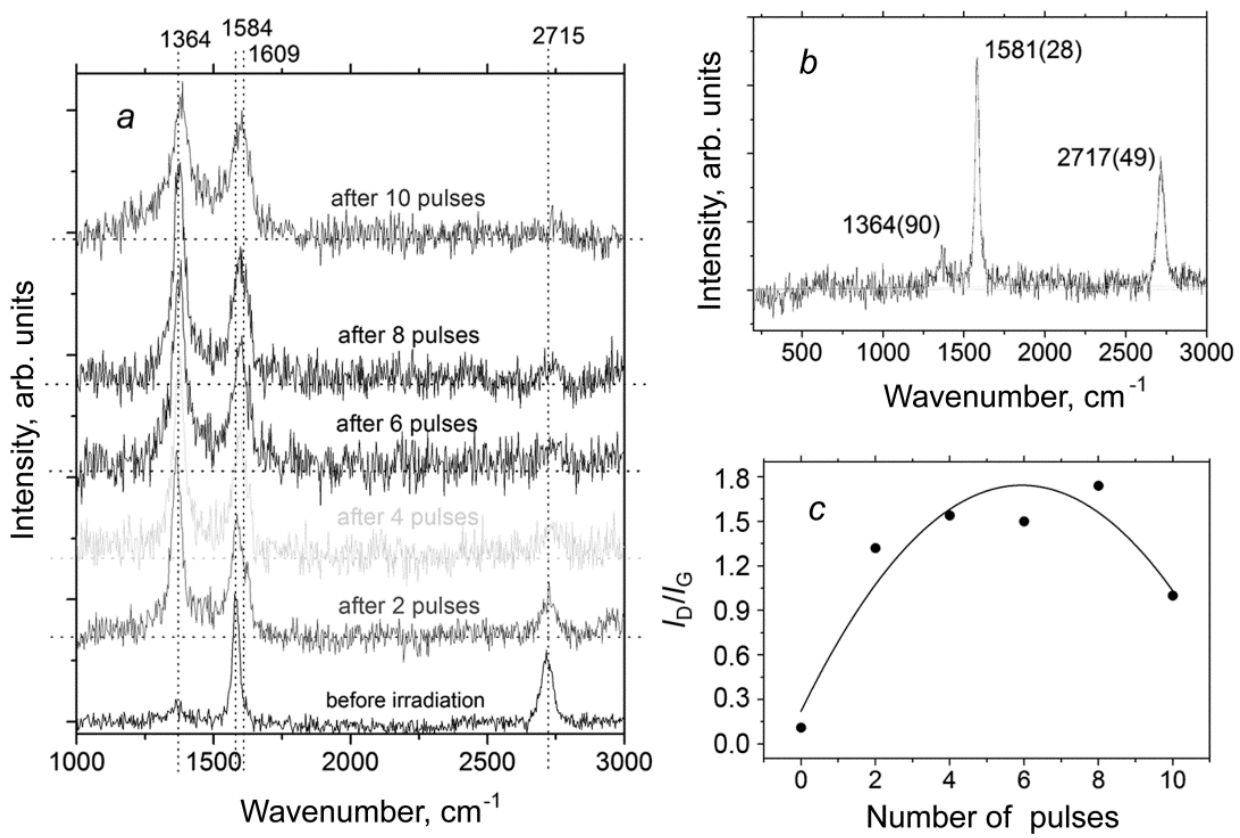

Fig. 2. Raman spectra of graphene depending on the number of ion pulses (the average absorbed dose). All spectra were normalized by the G-mode intensity $(a)$. The Raman spectrum of graphene before irradiation. Figures in the parentheses indicate the FWHM $(b)$. Dependence of the $I_{\mathrm{D}} / I_{\mathrm{G}}$ mode intensity ratio on the number of ion pulses (the average absorbed dose) (c).

the decrease of $I_{\mathrm{D}} / I_{\mathrm{G}}$. Analogous behavior of the $\mathrm{D}$ mode under irradiation by an ion beam was pointed out in [7]. The authors emphasized that the position of the $I_{\mathrm{D}} / I_{\mathrm{G}}$ maximum depended on the ratio of such characteristics, as the ion type (mass) and energy. Almost linear decrease of the 2D-mode intensity with increasing integral implanted dose can also be noted.

Based on results of theoretical analysis of defect formation in graphene film under IB irradiation presented in $\left[8\right.$, 9], we can conclude that irradiation with a $290 \mathrm{keV} \mathrm{C}\left(\mathrm{C}^{2+}\right)$ and $\mathrm{H}^{+}$ion beam with duration of $80 \mathrm{~ns}$ leads to minimal degradation of the free-standing graphene film that differs radically from the results presented above. For a more detailed analysis of the results, let us consider the interaction of carbon and hydrogen ions with a graphenebased target on a copper substrate. The accelerated particles, penetrating deep into the structure, are involved in complex processes of interaction with electrons and nuclei of the target material. The efficiency of these processes is characterized by the deceleration ability of the system $(\partial E / \partial x)_{e}$ and $(\partial E / \partial x)_{n}$ - energy losses due to interaction with nuclei and electronic subsystem per unit length. We note that a number of theoretical works (for example, see [15]) are devoted to theoretical modeling of PIB interaction with solid. Within the limits of that works, commercially accessible program SRIM-2013 (http://www.s rim.org/) was used to analyze in the first approximation the ion distributions in the examined target. Thus, according to our simulation for a pure copper target, $(\partial E / \partial x)_{e}$ and $(\partial E / \partial x)_{n}$ were $\sim 800$ and $\sim 50 \mathrm{keV} / \mu \mathrm{m}$ for interaction with $290 \mathrm{keV} \mathrm{\textrm {C } ^ { + }}\left(\mathrm{C}^{2+}\right)$ ions, respectively; in this case, the mean free paths of $\mathrm{C}^{+}\left(\mathrm{C}^{2+}\right)$ ions were $\sim 0.3 \mu \mathrm{m}$. For hydrogen ions $\mathrm{H}^{+}$, these values were 170 and $0.2 \mathrm{keV} / \mu \mathrm{m}$, respectively, and the mean free paths were $1.5 \mu \mathrm{m}$. Considering that the mean free paths of $\mathrm{H}^{+}$ions are twice as much as of $\mathrm{C}^{+}\left(\mathrm{C}^{2+}\right)$ ions and the elemental composition of the ion beam, we can conclude that the main contribution to the subsurface effects and as a result, to graphene degradation, comes from the interaction with $\mathrm{C}^{+}\left(\mathrm{C}^{2+}\right)$ ions. The distribution of $\mathrm{C}^{+}$ $\left(\mathrm{C}^{2+}\right)$ and $\mathrm{H}^{+}$ions in the copper foil target with thickness of $25 \mu \mathrm{m}$ and graphene film on its surface is shown in Fig. 3. The graphite parameters were used to model the processes of PIB interaction with the graphene target considering the 

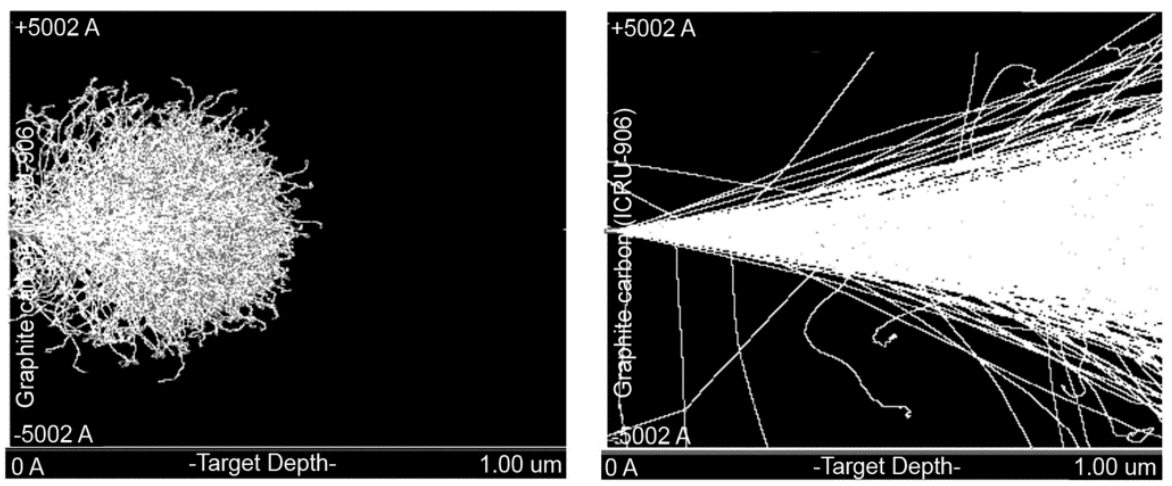

a
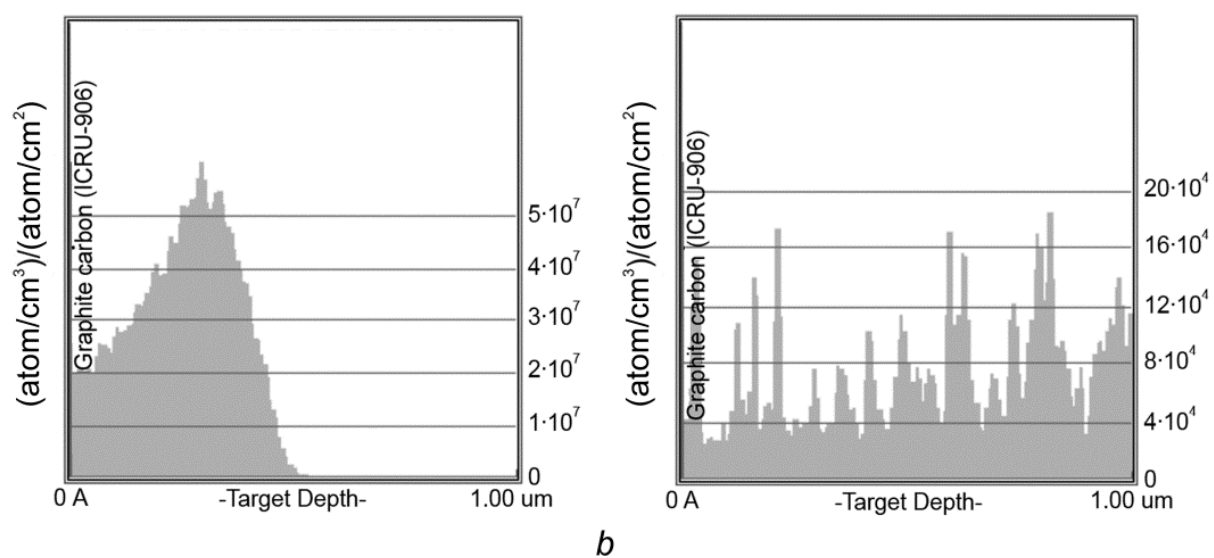

Fig. 3. Tracks of $\mathrm{C}^{+}$(left photograph) and $\mathrm{H}^{+}$ions (right photograph) in the copper target with graphene film on the surface $(a)$. Distribution of recoil atoms in the copper substrate irradiated by $\mathrm{C}^{+}$(left picture) and $\mathrm{H}^{+}$ions (right picture) $(b)$. The statistics was based on an analysis of 2000 ions.

difference between the binding energies of carbon atoms in graphene and graphite. The binding energy of carbon atoms was set equal to $22 \mathrm{eV}$. The thickness of the carbon layer was $3.4 \AA$. The thickness of the copper substrate was $25 \mu \mathrm{m}$.

Analyzing the distribution of $\mathrm{Cu}$ recoil atoms with depth of penetration of $\mathrm{C}^{+}\left(\mathrm{C}^{2+}\right)$ ions into the substrate, the localization of the cascades of recoil atoms (ions) near the surface can be noted. Moreover, such secondary atoms (ions) possess a lower energy, and according to the available data of theoretical analysis [8, 9], cause graphene degradation in the process of interaction.

As is well known, the thermal effects provide the basis for modification process under irradiation of a solid with pulsed charged particle beams. Therefore, it is important to consider additionally the dynamics of the copper surface temperature under PIB irradiation. As demonstrated in [16], without phase transitions, the solution of this problem is reduced to the solution of the heat equation

$$
\frac{\partial T}{\partial t}=k_{0} \frac{\partial^{2} T}{\partial x^{2}}+q(x, t), 0<x<1,0<t
$$

with initial and boundary conditions

$$
T(x, 0)=10<x<\left.1 \frac{\partial T}{\partial x}\right|_{x=0}=\left.\frac{\partial T}{\partial x}\right|_{x=1}=0, t>0 .
$$




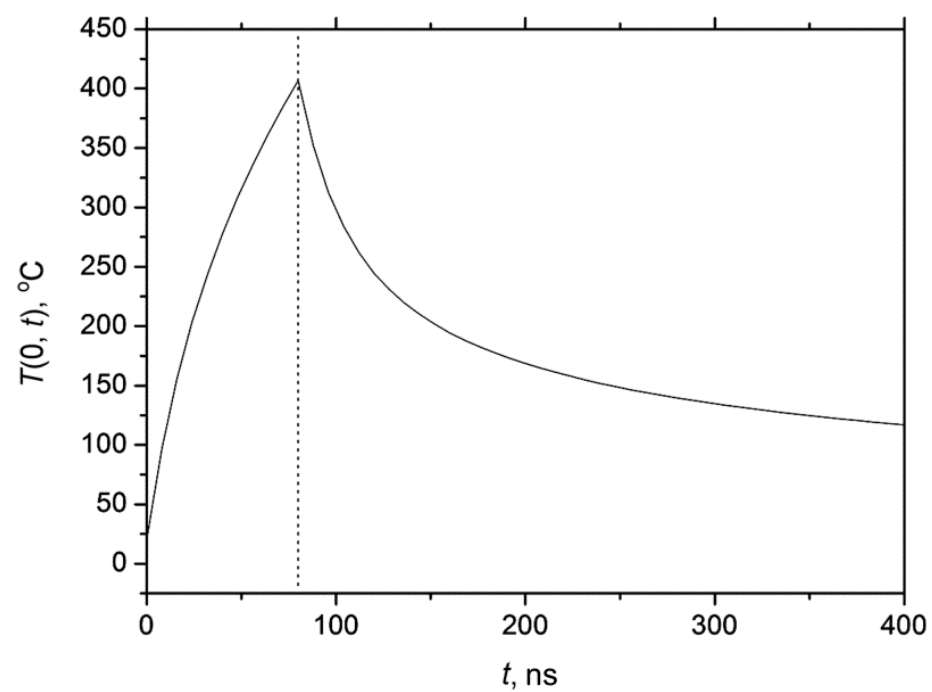

Fig. 4. Dependence of the surface temperature of the copper foil on the time of irradiation by one pulse of the beam current. The vertical straight line in the plot indicates the time of source switching off.

Here $T=T / T_{0}$ ( $T_{0}$ is the initial sample temperature $), x=x / l_{0}\left(l_{0}\right.$ is the sample thickness $), t=t / \tau_{0} \quad\left(\tau_{0}\right.$ is the pulse duration equal to $80 \mathrm{~ns}), k_{0}=\frac{\lambda \tau}{\rho c l_{0}{ }^{2}}$ ( $\lambda$ is the coefficient of thermal conductivity of copper $\left(400 \mathrm{~W} \cdot \mathrm{m}^{-1} \cdot \mathrm{K}\right), \rho$ is the copper density $\left(8900 \mathrm{~kg} \cdot \mathrm{m}^{-3}\right), c$ is the specific thermal capacity of copper $\left(400 \mathrm{~J} \cdot \mathrm{kg}^{-1} \cdot \mathrm{K}^{-1}\right), q(x, t)$ describes the influence of the charged particle beam $q(x, t)=q_{0} Q(x, t) \quad\left(q_{0}=E_{0} j_{\max } \tau / 2 Z e R_{0} c \rho T_{0}\right.$, where $R_{0}$ is the free path length of copper ions in the target, $E_{0}$ is the energy of ions, $j_{\max }$ is the ion current density, and the function $Q(x, t)$ describes the source shape and its influence, see [16]).

The time dependence of the copper foil surface temperature $(T(0, t))$ is shown in Fig. 4. As can be seen, irradiation by one pulse leads to heating of the copper surface up to no more than $500^{\circ} \mathrm{C}$. As noted above, the samples were irradiated with series of two pulses with the period between pulses $\sim 2 \mathrm{~s}$ that, in turn, was suffice for relaxation of the thermal effects. Considering that the CVD growth of graphene assumes temperatures $\sim 1000^{\circ} \mathrm{C}$, it is possible to conclude that during our experiment, the thermal effects caused by BIP irradiation can be neglected, and the main contribution comes from the effects of recoil atoms.

\section{CONCLUSIONS}

From the analysis of the results presented above, the significant influence of the substrate on the radiation resistance of graphene can be indicated. In this context, one of the important engineering problems in the development of modern on-chip optoelectronic devices based on graphene capable effectively operate under conditions of enhanced radiation loading, is the choice of the optimal substrate whose contribution to the degradation of functional graphene properties will be minimal.

The authors express their gratitude to K. G. Batrakov (Research Institute for Nuclear Problems of Belarusian State University) for useful discussions.

This work was supported in part by the Russian Foundation for Basic Research (Project No. 17-38-50050) and by the Program H2020 (Project MSCA-RISE-2014 ID 644076 CoExAN). 


\section{REFERENCES}

1. V. Singh, D. Joung, L. Zhai, et al., Prog. Mater. Sci., 56, 1178-1271 (2011).

2. C. Lee, X. Wei, J. W. Kysar, and J. Hone, Science, 321, 385 (2008).

3. K. S. Novoselov, A. K. Geim, S. V. Morozov, et al., Nature, 438, 197-200 (2005).

4. A. A. Balandin, S. Ghosh, W. Bao, et al., Nano Lett., 8, 902-907 (2008).

5. S. S. Dhillon, M. S. Vitiello, E. H. Linfield, et al., J. Phys. D, 50, 043001 (2017).

6. I. A. Tsyganov, N. P. Guseinov, and A. M. Il'in, Izv. Vyssh. Uchebn. Zaved. Fiz., 54, No. 1/3, 307-310 (2011).

7. G. Gawlik, P. Ciepielewski, J. Jagielski, and J. Baranowski, Nucl. Instrum. Methods Phys. Res. B, 406, 683688 (2017).

8. O. Lehtinen, J. Kotakoski, A. V. Krasheninnikov, et al., Phys. Rev. B, 81, 153401 (2010).

9. O. Lehtinen, J. Kotakoski, A. V. Krasheninnikov, and J. Keinonen, Nanotechnology, 22, 175306 (2011).

10. S. Stolyarova, D. Stolyarov, K. Bolotin, et al., Nano Lett., 9, 332-337 (2009).

11. I. Vlassiouk, M. Regmi, P. Fulvio, et al., ACS Nano, 5 (7), 6069-6076 (2011).

12. A. Ferrari, J. Meyer, V. Scardaci, et al., Phys. Rev. Lett., 97, 187401-4 (2006).

13. S. U. Yu, Y. Cho, B. Park, et al., Chem. Commun., 49, 5474 (2013).

14. Y. Hao, Y. Wang, L. Wang, et al., Small, 6 (2), 195-200 (2010).

15. V. I. Boiko and V. V. Evstigneev, Introduction to Physics of Interaction of High-Power Charged Particle Beams with Matter [in Russian], Energoatomizdat, Moscow (1988).

16. I. V. Amirkhanov, E. V. Zemlyunaya, I. V. Puzynin, et al., Report of Joint Institute of Nuclear Research R112001-164, Dubna (2001). 